\author{
Military Technical College \\ Kobry El-Kobbah, \\ Cairo, Egypt.
}

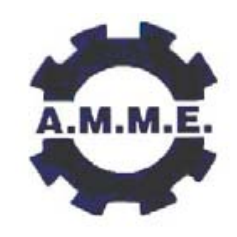

\title{
DYNAMICS AND STABILITY OF GUN-BARRELS WITH MOVING BULLETS
}

\author{
WAGIH $^{*}$ A., ALADIN ${ }^{* *}$ M., TAWFIK ${ }^{* * *}$ M. and NEGM ${ }^{* * *}$ H.M.
}

\begin{abstract}
In this study, the stability of a Timoshenko beam under the effect of a moving projectile will be reintroduced using simple eigenvalue analysis of a finite element model. The eigenvalues of the beam change with the mass, speed, and position of the projectile, thus, the eigenvalues are evaluated for the system with different speeds and masses at different position until the lowest eigenvalue reaches zero indicating the instability occurrence. Then the dynamics of the beam will be investigated using the Newmark algorithm at different values of speed and mass ratios. It is concluded that the technique used to predict the stability boundaries is simple, accurate, and reliable.
\end{abstract}

\section{KEY WORDS}

Timoshenko beams, beam dynamics, beam stability, time response, cantilever beam and moving bullet

** Egyptian Air-force, Cairo, Egypt.

** Modeling and Simulation in Mechanics, German University in Cairo.

${ }_{* * *}^{*}$ Mechanical Engineering Department, British University in Egypt, Cairo, Egypt.

**** Aerospace Engineering Department, Cairo University, Cairo, Egypt. 


\section{NOMENCLATURE}

A cross-sectional area

[ $\left.C_{b s l l t t}\right]$ Coriolis damping matrix

E beam Young's modulus

Ubullat $\}$ bullet weight vector

G shear modulus

$g \quad$ acceleration due to gravity

I beam moment of inertia

$i \quad$ element number

$K_{s} \quad$ shear correction factor

$\left|K_{\text {benaivg }}\right| \quad$ beam bending- stiffness matrix

$\left[K_{\text {shear }}\right] \quad$ beam shear-stiffness matrix

$\left[K_{\text {bullet }}\right] \quad$ bullet stiffness matrix

$L \quad$ beam length

$l \quad$ element length

$\left\lfloor M_{\text {bending }}\right\rfloor \quad$ beam bending-mass matrix

$\left[M_{\text {shear }}\right] \quad$ beam shear-mass matrix

$\left[M_{\text {bullet }}\right]$ bullet mass matrix

m bullet mass

$N \quad$ shape function

$T \quad$ total kinetic energy of beam system

$t \quad$ time

$\Delta t \quad$ time step

U total potential energy of beam system

V bullet speed

$W_{\text {bullat }} \quad$ total work done by the bullet 


$\begin{array}{ll}w & \text { transverse displacement of the beam } \\ w_{b} & \text { transverse displacement of the beam due to bending } \\ w_{s} & \text { transverse displacement of the beam due to shear } \\ w_{i}^{e} & \text { nodal degree of freedom } \\ w_{\text {nomalized }} & \text { normalized deflection }\left(=w /\left(\frac{\rho A L_{g}}{E_{L} / L^{\mathrm{B}}}\right)\right) \\ x_{m} & \text { position of the bullet }\end{array}$

\section{GREEK SYMBOLS}

$\begin{array}{ll}u & \text { dimensionless mass ratio }(=m L / \mu A L) \\ \rho & \text { dimensionless speed ratio }(=V L /(2 \pi \sqrt{\rho A / E I})) \\ \delta & \text { Dirac delta function } \\ \rho & \text { mass density of the beam }\end{array}$

\section{INTRODUCTION AND LITERATURE SURVEY}

The problem of the dynamics and stability of beams carrying moving masses drew a lot of attention in the past half century due to the applications that require it such as fast trains, motion on bridges, and light guns mounted on aircraft. In 1971, Nelson and Conover [1]presented a study of the problem of an infinite thin beam with periodically distributed simple supports resting on elastic foundation with a train of masses moving on it at constant speed. They applied Galerkin method to the proposed approximate solution to get the system equations then applied the Floquet theorem to get the stability boundaries for the periodically repeating system. Simultaneously, Benedetti [2] and [3] used a similar approach and could present an analytical relation between the mass parameter and the critical speed parameter using classical techniques.

Since, most of the research was directed to civil structures and lathe-machined work pieces, the stability regions were not of major interest for general structures. Rather, the response of the structure to moving loads or masses presented a more practical problem. Further, most of the studies were interested in problems with periodically supported beams that simulate train rails, see [4], [5], [6], and [7] as examples for such studies.

Recently, the emergence of the need for very light guns that are mounted on combat aircraft reintroduced the stability problem with new conditions. The motion of the bullets inside the gun barrels introduces compression force on the shell walls, in turn; this compression may cause dynamic buckling [2] and excessive vibration in the shell wall [8]. This type of instability, though of major importance, will not be covered in this study. 
The problem of projectiles inside gun barrels, though of important application, was not the subject of many researches. In [9] and [10], the stability problem was studied using the impulsive parametric excitation theory [11] [12] for a thin beam with periodically distributed controllers. The study ignored the effect of the dynamics of the barrel shell. The problem of the dynamics of the barrel shell under the effect of the moving projectile with shock and expansion waves was studied in [8] but the study did not tackle the stability problem. In [13], one of the very rare studies that handled finite beams was presented. In that study, the beam under investigation was modeled as a Timoshenko beam with simple supports and elastic foundation. The results presented different cases of multiple masses and foundation stiffness but did not present any comparison with published or experimental results. Recently, [14] presented an algorithm for the stability analysis of gun barrels modeled as an Euler-Bernoulli beam.

In this study, the stability of a Timoshenko beam under the effect of a moving projectile will be reintroduced using simple eigenvalue analysis of a finite element model. The eigenvalues of the beam change with the mass, speed, and position of the projectile, thus, the eigenvalues are evaluated for the system with different speeds and masses at different position until the lowest eigenvalue reaches zero indicating the instability occurrence. Then the dynamics of the beam will be investigated using the Newmark algorithm at different values of speed and mass ratios.

\section{MODEL}

The model derived in this section will have the following assumptions; the gun barrel will be modeled as thick beam that follows the Timoshenko beam theorem and the barrel deflections is small. The total displacement may be presented as [15-16]

$$
w(x)=w_{b}(x)+w_{s}(x)
$$

The element is assumed to have two nodes with 4 degrees of freedom per node. As shown in Fig.1. the degrees of freedom are the bending displacement, bending slope, shear displacement and shear slope. The subscript $b$ and $s$ denote the bending and shear degrees of freedom respectively. Both bending and shear deformations are assumed to follow a third order polynomial. The element displacement function can be written as:

$$
w_{i}(x)=\left\lfloor N_{1}(x) \quad N_{2}(x) \quad N_{3}(x) \quad N_{4}(x)\right\rfloor \times\left\lfloor\begin{array}{llll}
w_{i 1} & w_{i 1}^{\prime} & w_{i 2} & w_{i 2}^{\prime}
\end{array}\right]^{T}
$$

or:

$$
w_{i}(x)=\lfloor N(x)\rfloor\left\{w_{i}^{e}\right\}
$$

where $N(x)$ is the shape function and $w_{i}$ denotes either $w_{b}$ or $w_{s}$. The Hamilton principle states that:

$$
\int_{t_{1}}^{t_{2}} \delta \Pi d t=\int_{t_{1}}^{t_{2}} \delta\left(T-U+W_{\text {ballet }}\right) d t=0
$$


where $T$ is the kinetic energy, $U$ is the potential energy, and $W_{b u l l e t}$ is the work done by the bullet on the beam. The kinetic energy of the system may be written as

$$
T=\frac{1}{2} \int_{0}^{l} \rho A\left(\dot{w}_{b}+\dot{w}_{s}\right)^{2} d x+\frac{1}{2} m\left(\left(\dot{w}_{b}+\dot{w}_{s}\right)^{2}+2 V\left(\dot{w}_{b}+\dot{w}_{s}\right)\left(\frac{\partial w_{b}}{\partial x}+\frac{\partial w_{s}}{\partial x}\right)\right)_{x=x_{m}}
$$

where $\rho$ is the beam mass density, $A$ is the beam cross section area, $m$ is the bullet mass, $V$ is the bullet speed, $w_{b}$ and $w_{s}$ are the beam bending and shear displacement, and $x_{m}$ is the position of the bullet. The potential energy of the system may be written as

$$
U=\frac{1}{2} \int_{0}^{l}\left(E I\left(\frac{\partial^{2} w_{b}}{\partial x^{2}}\right)^{2}+G A K_{s}\left(\frac{\partial^{2} w_{s}}{\partial x^{2}}\right)^{2}\right) d x-m g\left(w_{b}+w_{s}\right)_{x=x_{m}}
$$

where $E$ is the beam modulus of elasticity, $l$ is the beam second moment of area $G$ is the modulus of rigidity, $A$ is the cross-sectional area, $K_{s}$ is the shear factor which is taken equal to 1 , and $g$ is the gravitational acceleration. And the work done by the bullet may be written as

$$
W_{\text {bullet }}=\frac{1}{2}\left(m V^{2}\left(\frac{\partial w_{b}}{\partial x}+\frac{\partial w_{s}}{\partial x}\right)^{2}\right)_{x=x_{m}}
$$

The variation of the kinetic energy may be written as

$$
\begin{aligned}
\delta T= & \int_{0}^{l} \rho A\left(\delta \dot{w}_{b}+\delta \dot{w}_{s}\right)\left(\dot{w}_{b}+\dot{w}_{s}\right) d x+ \\
& m\left(\left(\delta \dot{w}_{b}+\delta \dot{w}_{s}\right)\left(\dot{w}_{b}+\dot{w}_{s}\right)+V\left(\delta \dot{w}_{b}+\delta \dot{w}_{s}\right)\left(\frac{\partial w_{b}}{\partial x}+\frac{\partial w_{s}}{\partial x}\right)+V\left(\dot{w}_{b}+\dot{w}_{s}\right)\left(\frac{\partial \delta w_{b}}{\partial x}+\frac{\partial \delta w_{s}}{\partial x}\right)\right)_{x=x_{m}}
\end{aligned}
$$

And the variation of the potential energy may be written as

$$
\delta U=\int_{0}^{l}\left(E I\left(\frac{\partial^{2} \delta w_{b}}{\partial x^{2}}\right)\left(\frac{\partial^{2} w_{b}}{\partial x^{2}}\right)+G A K_{s}\left(\frac{\partial^{2} \delta w_{s}}{\partial x^{2}}\right)\left(\frac{\partial^{2} w_{s}}{\partial x^{2}}\right)\right) d x-m g\left(\delta w_{b}+\delta w_{s}\right)_{x=x_{m}}
$$

While the variation of the work done by the bullet may be written as 


$$
W_{\text {bullet }}=\left(m V^{2}\left(\frac{\partial \delta w_{b}}{\partial x}+\frac{\partial \delta w_{s}}{\partial x}\right)\left(\frac{\partial w_{b}}{\partial x}+\frac{\partial w_{s}}{\partial x}\right)\right)_{x=x_{m}}
$$

Applying the Hamilton principle, we may write the element equation of motion as:

$$
\begin{aligned}
& \left.\left(\left[\begin{array}{cc}
{\left[M_{\text {bending }}\right]+\left[M_{\text {shear }}\right]} & {\left[M_{\text {bending }}\right]} \\
{\left[M_{\text {bending }}\right]} & {\left[M_{\text {bending }}\right]}
\end{array}\right]+\left[\begin{array}{ll}
{\left[M_{\text {bullet }}\right]} & {\left[M_{\text {bullet }}\right]} \\
{\left[M_{\text {bullet }}\right]} & {\left[M_{\text {bullet }}\right]}
\end{array}\right]\right)\left\{\begin{array}{l}
\ddot{w}_{b} \\
\ddot{w}_{s}
\end{array}\right\}+\left[\begin{array}{ll}
{\left[C_{\text {bullet }}\right]} & {\left[C_{\text {bullet }}\right]} \\
{\left[C_{\text {bullet }}\right]} & {\left[C_{\text {bullet }}\right]}
\end{array}\right]\right)\left\{\begin{array}{l}
\dot{w}_{b} \\
\dot{w}_{s}
\end{array}\right\}+ \\
& \left(\left[\begin{array}{cc}
{\left[K_{\text {bending }}\right]} & 0 \\
0 & {\left[K_{\text {shear }}\right]}
\end{array}\right]-\left[\begin{array}{cc}
{\left[K_{\text {bullet }}\right]} & {\left[K_{\text {bullet }}\right]} \\
{\left[K_{\text {bullet }}\right]} & {\left[K_{\text {bullet }}\right]}
\end{array}\right]\right)\left\{\begin{array}{l}
w_{b} \\
w_{s}
\end{array}\right\}=\left\{\begin{array}{l}
f_{w} \\
f_{w}
\end{array}\right\}+\left\{\begin{array}{l}
f_{\text {bullet }} \\
f_{\text {bullet }}
\end{array}\right\}
\end{aligned}
$$

where the beam mass matrices are evaluated by:

$$
\begin{gathered}
{\left[M_{\text {bending }}\right]=\int_{0}^{l} \rho A\{N(x)\}^{T}\{N(x)\} d x} \\
{\left[M_{\text {shear }}\right]=\int_{0}^{l} \rho I\left\{N_{x}(x)\right\}^{T}\left\{N_{x}(x)\right\} d x}
\end{gathered}
$$

where $N(x)$ are the beam interpolation polynomials. The effective bullet mass matrix is evaluated at the bullet position as

$$
\left[M_{\text {bulle: }}\right]=m\{N(x)\}^{T}\{N(x)\}_{w=w_{m}}
$$

While the beam stiffness matrices are evaluated by

$$
\left[K_{\text {bending }}\right]=\int_{0}^{l} E I\left\{N_{x x}(x)\right\}^{T}\left\{N_{x x}(x)\right\} d x
$$

and

$$
\left[K_{\text {shear }}\right]=\int_{0}^{l} G A K_{s}\left\{N_{x}(x)\right\}^{T}\left\{N_{x}(x)\right\} d x
$$

The effective geometric bullet stiffness matrix is evaluated at the bullet position as

$$
\left[K_{\text {bullet }}\right]=\left.m V^{2}\left\{N_{x}(x)\right\}^{T}\left\{N_{x}(x)\right\}\right|_{x=x_{m}}
$$

The effective bullet Coriolis matrix is evaluated by 


$$
\left[C_{\text {bullet }}\right]=\left.2 m V(N(x)]^{\mathrm{T}}\left(N_{x}(x)\right]\right|_{x=x_{m}}
$$

And the forces due to the bullet weight is evaluated by

$$
\left\{f_{\text {bullot }}\right\}=-\left.\{N(x)\}^{T} m g\right|_{x=x_{m}}
$$

\section{Stability Boundaries}

To obtain the eigenvalues of the system, including the effect of all components, we will need to transform the system into a first order system by the standard transformation below [14]

$$
z=\dot{w}
$$

Using the above transformation, we will obtain the equation of motion in the form

$$
\left\{\begin{array}{l}
W \\
\dot{z}
\end{array}\right\}=\left[\begin{array}{cc}
0 & I_{n \times n} \\
-[M]^{-1}[K] & -[M]^{-1}[C]
\end{array}\right]\left\{\begin{array}{l}
W \\
z
\end{array}\right\}
$$

where:

$$
\begin{aligned}
& \left.[M]=\left(\begin{array}{cc}
{\left[M_{\text {bending }}\right]+\left[M_{\text {shear }}\right]} & {\left[M_{\text {bending }}\right.} \\
{\left[M_{\text {bending }}\right]} & {\left[M_{\text {bending }}\right.}
\end{array}\right]+\left[\begin{array}{ll}
{\left[M_{\text {bullet }}\right]} & {\left[M_{\text {bullet }}\right]} \\
{\left[M_{\text {bullet }}\right]} & {\left[M_{\text {bullet }}\right]}
\end{array}\right]\right) \\
& {[K]=\left(\left[\begin{array}{cc}
{\left[K_{\text {bending }}\right]} & 0 \\
0 & {\left[K_{\text {shear }}\right]}
\end{array}\right]-\left[\begin{array}{ll}
{\left[K_{\text {bullet }}\right]} & {\left[K_{\text {bullet }}\right]} \\
{\left[K_{\text {bullet }}\right]} & {\left[K_{\text {bullet }}\right.}
\end{array}\right]\right)} \\
& {[C]=\left[\begin{array}{ll}
{\left[C_{\text {bullet }}\right]} & {\left[C_{\text {bullet }}\right.} \\
{\left[C_{\text {bullet }}\right]} & {\left[C_{\text {bullet }}\right.}
\end{array}\right]}
\end{aligned}
$$

For the above system, the eigenvalues should represent the natural frequencies of oscillation of the beam with the bullet. The eigenvalues should all be complex with nonzero imaginary parts for all values of the speed that are below the critical speed. As the bullet speed reaches the critical speed, the smallest complex pair will have zero imaginary parts. 
The search for the critical values of the speed may be done using the following algorithm

1- Select the bullet mass

2- Select the bullet speed

3- Change the value of the $x$-location of the bullet and evaluate the eigenvalues of the system

4- If all eigenvalues have non-zero imaginary parts then increases speed and go to step 3 else go to step 6

5- If all speed values did not reach the critical value then reset speed value and increase mass up to a given limit and proceed to step 3. If mass limit is reached terminate

6- Store the values of the critical speed and the mass

7- Increase speed up to a given limit and proceed to step 3. If mass limit is reached terminate

The above procedure may be repeated for all values of mass, speed, and boundary conditions and different information may be compiled out of the extracted data.

\section{Time Response}

The time response of the system presented by equation (11) may be obtained using the Newmark algorithm [17] as follows:

$$
\left.[M]\{w\}+[C][w\}+[K]\{w\}=U_{w}\right\}
$$

1- Evaluate the acceleration of the system using

$$
\begin{gathered}
\left([M]+\delta A t[C]+\beta A t^{2}[K]\right)[\ddot{w}\}_{i+\Delta L} \\
=\left\{f_{w}\right\}_{t+\Delta t}-[C]\left(\{\dot{w}\}_{t}+(1-\delta) \Delta t\{\ddot{w}\}_{t}\right)-[K]\left(\{w\}_{t}+\Delta t\{\dot{w}\}_{\tau}+\left(\frac{1}{2}-\mu\right) \Delta t^{2}\{\ddot{w}\}_{t}\right)
\end{gathered}
$$

2- Then the velocity and displacement may be evaluated using

$$
\begin{aligned}
& \{\dot{w}\}_{t+\Delta t}-\{\dot{w}\}_{t}+(1-\delta) \Delta l\{\dot{w}\}_{t}+\mu \Delta l\{\dot{w}\}_{t+\Delta t} \\
& \{w\}_{t+\Delta t}-\{w\}_{t}+\Delta t\{\dot{w}\}_{t}+\left(\frac{1}{2}-\mu\right) \Delta t^{2}\{\dot{w}\}_{t}+\mu \Delta t^{2}\{\ddot{w}\}_{t}
\end{aligned}
$$

In the above algorithm, $\delta$ and $\mu$ are parameters that have to obey the constraints $\delta \geq 0.5$ and $\mu \geq \frac{1}{4}\left(\delta+\frac{1}{2}\right)^{2}$. 


\section{RESULTS AND DISCUSSION}

\section{Stability Boundaries}

A program was written using MATLAB to perform the calculations of the problem using the following data; Beam modulus of elasticity $71 \mathrm{GPa}$, Density $2840 \mathrm{~kg} / \mathrm{m}^{3}$, inner radius $0.007 \mathrm{~m}$, and the shell thickness takes one of two values $(1 \mathrm{~mm}$ and $10 \mathrm{~mm}$ ). The projectile mass ranged from 0.005 to $0.75 \mathrm{Kg}$, and its speed ranged from 2.5 to $600 \mathrm{~m} / \mathrm{s}$. The program used 10 beam elements, see Ref [18], and checked for the eigenvalues at 20 equidistant points in each element. The convergence of the solution was checked and it was found that the 10 elements with 20 internal points gave adequate accuracy.

Figure 2 shows the results of the stability boundaries using the current model, Timoshenko beam model, compared to the results obtained by [14], Euler-Bernoulli beam model, with barrel thickness of $1 \mathrm{~mm}$. Where $\alpha=m / \rho A L$ is the mass parameter and $\beta=V L /(2 \pi \sqrt{\rho A / E I})$ is the velocity parameter. It is evident from the graph that the results of the Timoshenko model are more conservative, softer beam, which is consistent with the fact that the model allows for sheer deflections. Note that the regression formula obtained in [14] for the relation between $\alpha$ and $\beta$ is given by:

$$
\beta_{c,} l_{E-B \text { Model }} \approx 0.21 \alpha
$$

While the one obtained using the Timoshenko model is given by:

$$
\beta_{\text {mima. Madei }} \approx 0.124 \alpha^{-0.54}
$$

It has to be noted at this step that the results obtained for a gun barrel with shell thickness of $10 \mathrm{~mm}$ is given by:

$$
\left.\beta_{\text {er }}\right|_{\text {Timo. Model }} \propto 0.124 \alpha^{-0.55}
$$

Which is essentially the same regression formula. This result indicates that the finite element model used in this paper does not suffer from sheer locking phenomenon in the range of geometry used.

\section{Time Response}

A program was developed for the time response of the barrel to moving bullets using the Newmark technique presented earlier and the results were compared to response presented in Ref. [19] with the consideration that the reference did not include the effect of the external work done by the bullet on the barrel. The values used for the algorithm 
parameters were $\delta=0.52$ and $\mu=0.27$. In all numerical results for the dynamics, presented in this section, the time step used was $1 / 2400$ of the total time required for the bullet to transverse the barrel. Convergence of the solution was tested using different values of the time step and it was found that this value was accurate and convenient.

The response of the barrel to the motion of the bullet is one of the important aspects that should be considered when designing a gun-barrel as shown in Fig.3. As the instability described in the previous section is a static instability, pitchfork bifurcation, which is reflected in higher dynamic response to the external excitation, rather than self-excited vibrations that are associated with Hopf bifurcations. The higher the response becomes, the more the time between the bullets should be to ensure the accuracy of target hits. The normalized deflection is calculated as:

$$
w_{\text {nomalized }}=w /\left(\frac{\rho A L g}{E I / L^{2}}\right)
$$

This normalization of the deflection will ensure the same response curve for the same values of $\alpha$ and $\beta$ regardless of the beam properties and geometry.

Figures 4 and 5 show the beam tip deflection due to bending deformation and due to shear deformation separately. It is obvious that the effect of the shear deformation is so small, specially for thin beams(i.e. Euler-Bernoulli beam).

\section{CONCLUSIONS}

In this study, a finite element model was used to predict the stability boundaries for beams modeled using Timoshenko beam theory with moving masses. An empirical relation between the mass parameter and critical speed parameter could be obtained for the cantilever beams given by the relation $\beta_{a .} \approx 0.124 \alpha^{-0.54}$ which was compared to the results obtained by an Euler-Bernoulli beam model.

Further, the model was used to predict the response of the tip of the gun-barrel to the motion of the bullet using Newmark algorithm. The results indicated that the neglection of the barrel mass changes the dynamic response characteristics and amplitude completely, thus, it is recommended that the barrel mass should not be ignored in any future studies.

\section{REFERENCES}

[1] Nelson, H. D. and Conover, R. A., "Dynamic stability of a beam carrying moving masses", Journal of Applied Mechanics, Vol. 38, pp. 1003-1006, (1971).

[2] Benedetti, G. A., "Transverse vibration and stability of a beam subject to moving mass loads", (1973).

[3] Benedetti, G. A., "Dynamic stability of a beam loaded be a sequence of moving mass particles", Journal of Applied Mechanics, Vol. 41, pp. 1069-1071, (1974). 
[4] Foda, M. A. and Abdul jabbar, Z.,"A dynamic function formulation for the response of a beam structure to a moving mass", Journal of Sound and Vibration, Vol. 210, pp. 295-306, (1998).

[5] Ichikawa, M., Miyakawa, Y. and Matsuda, A.,"Vibration analysis of continuous beam subjected to moving mass", Journal of Sound and Vibration, Vol. 230, pp. 493-506, (2000).

[6] Katz, R., et al.,"Dynamic Stability and Response of a beam subject to deflection dependent moving load", Journal of Vibration, Acoustics, Stress, and Reliability in Design, Vol. 109, pp. 361-365, (1987).

[7] Lin, Y. H. and Trethewey, M. W.,"Finite element analysis of elastic beams subjected to moving dynamic loads", Journal of Vibration and Acoustics, Vol. 112, pp. 323-342, (1990).

[8] Ruzzene, M. and Baz, A.,"Response of periodically stiffened shells to moving projectile propelled by internal pressure wave", Mechanics of Advanced Materials ans Structures, Vol. 13, pp. 267-284, (2006).

[9] Aldraihem, O. J. and Baz, A., "Dynamic Stability of Stepped Beams Under Moving Loads", Journal of Sound and Vibration, Vol. 250, pp. 835-848, (2002).

[10] Aldraihem, O. J. and Baz, A., "Moving-Loads-Induced Instability in Stepped Tubes", Journal of Vibration and Control, Vol. 10, pp. 3-23, (2004).

[11] Hsu, C. S., "Impulsive parametric excitation: Theory", Journal of Applied Mechanics, Vol. 39, pp. 551-558, (1972).

[12] Hsu, C. S. and Cheng, W. H., "Application of the theory of impulsive parametric excitation and new treatments of general parametric excitation problems", Journal of Applied Mechanics, Vol. 40, (1973).

[13] Makhertich, S., "Dynamic stability of a beam excited by a sequence of moving mass particles", Journal of the Acoustical Society of America, Vol. 115, pp. 1416-1419, (2004).

[14] Tawfik, Mohammad.,"Dynamics and Stability of Stepped Gun-Barrels with Moving Bullets", Advances in Acoustics and Vibration, Vol. In Press, (2008).

[15] Rao, S. and Gupta, R., "Finite element vibration analysis of rotating Timoshenko beams", Journal of Sound and Vibration, Vol. 242, pp. 103-124, (2001).

[16] Alaa El-Din, Maged and Tawfik, Mohammad.,"Vibration Attenuation in A Periodic Rotating Timoshenko Beam", Cairns : s.n., ICSV14, (2007).

[17] Krenk, Steen.,"Energy conservation in Newmark-based time integration algorithms", Computer Methods in Applied Mechanics and Engineering, Vol. 195, pp. 6110-6124, (2006).

[18] Alaa El-Din, Maged and Tawfik, Mohammad., "Vibration Attenuation in Rotating Beams with Periodically Distributed Piezoelectric Controllers", Vienna, Austria: s.n., 13th International Congress on Sound and Vibration, (2006).

[19] Parhi, D. R. and Behera, A. K., "Dynamic deflection of cracked beam with moving mass", Proceeding of the Institution of Mechanical engineering, Vol. 211, pp. 77-87, (1997). 


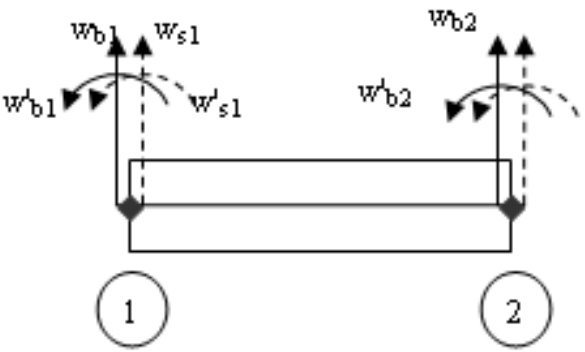

Fig. 1. Element Degrees of Freedom

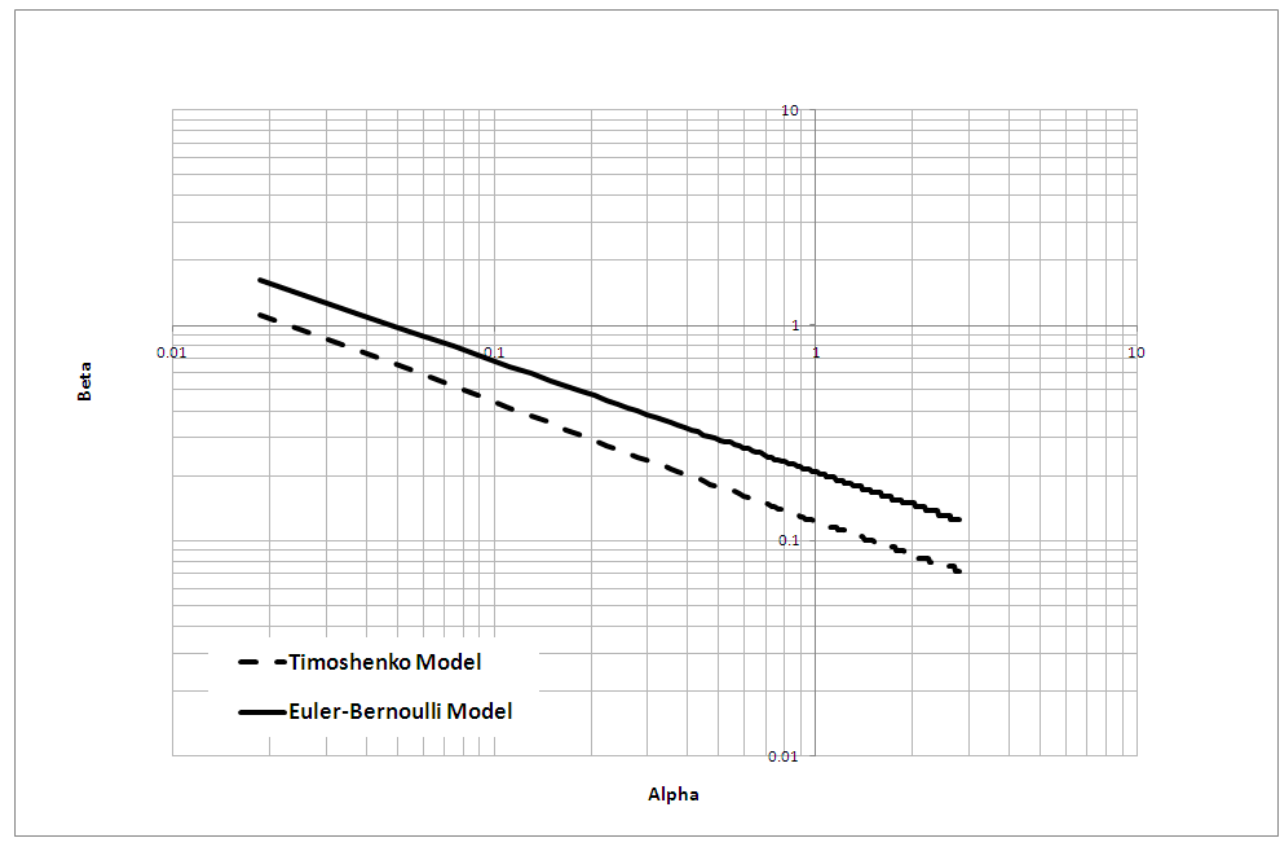

Fig. 2. Stability Boundaries for the gun barrel using two different beam models. 


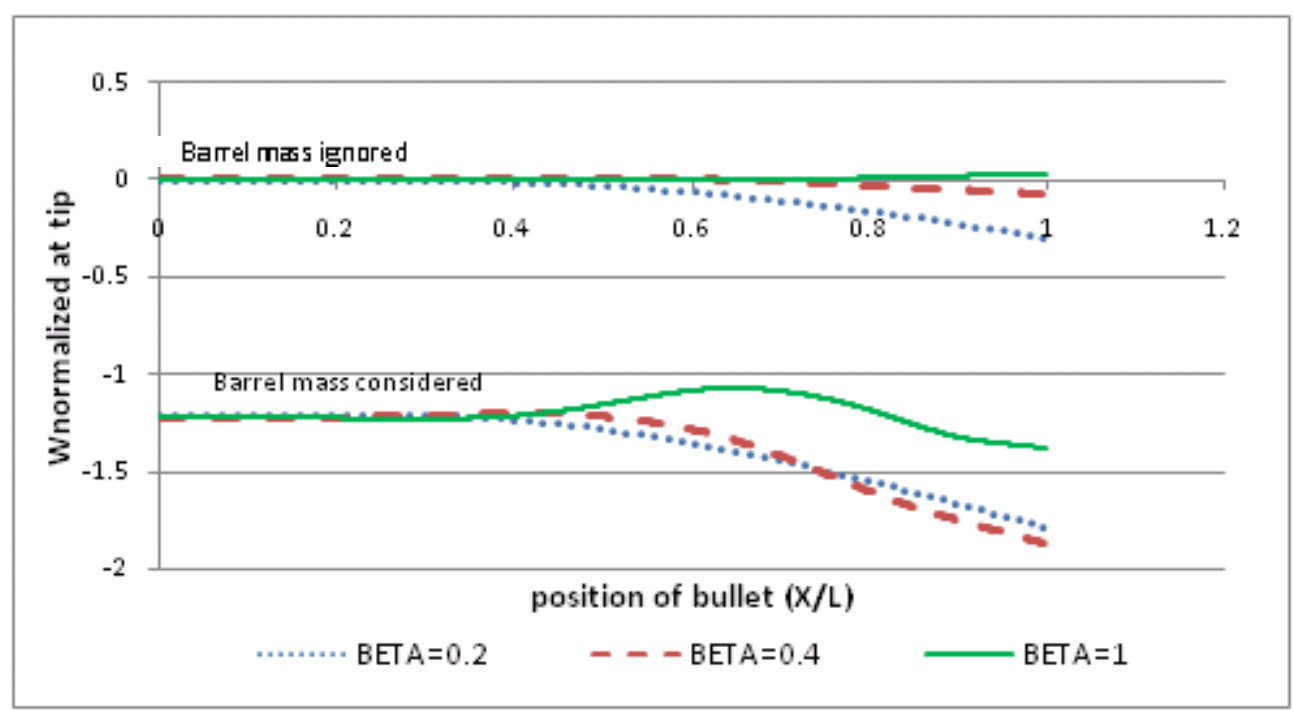

Fig. 3. Response of the barrel's tip vs. bullet position with and without considering the barrel's weight for $\alpha=0.2$ and different values of $\beta$

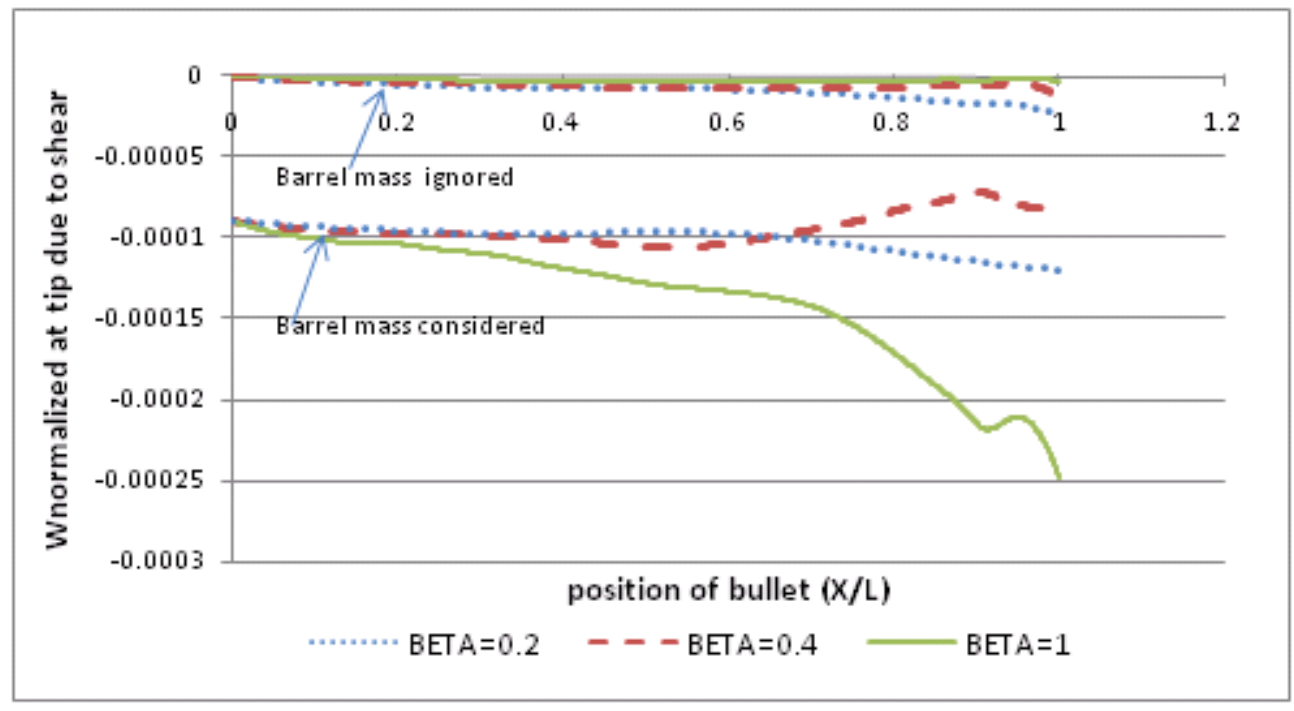

Fig. 4. Response of the barrel's tip (due to shear) vs. bullet position with and without considering the barrel's weight for $\alpha=0.2$ and different values of $\beta$ 


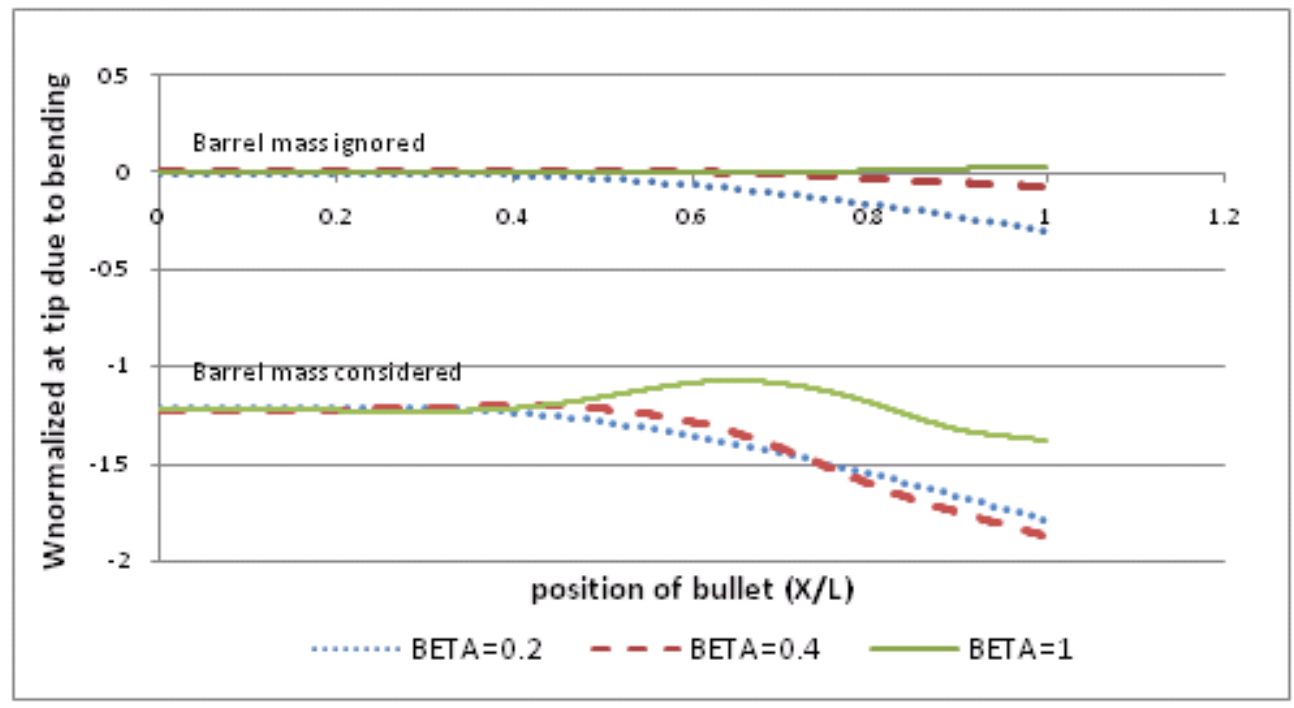

Fig. 5. Response of the barrel's tip (due to bending) vs. bullet position with and without considering the barrel's weight for $\alpha=0.2$ and different values of $\beta$ 